\title{
Study of Expert Control Systems for Power Transmission Line Inspection Robot
}

\author{
Xiao Hong ${ }^{a}$, Hao Luo ${ }^{b}$, Biwu Yan ${ }^{c}$, Lei Zheng ${ }^{d}$ \\ Wuhan NARI Limited Liability Company of State Grid Electric Power Research Institute, SGCC, \\ Wuhan, Hubei, 430074 \\ axiaohong.seu@qq.com, 'lhsgepri@163.com, ${ }^{c} 11318443 @ q q . c o m,{ }^{d}$ chenylei1218@163.com
}

Keywords: power transmission line, inspection robot, expert system

Abstract: The structure of mechanical and control system for power transmission line inspection robot was introduced. Then a new control method and its real-time implementation based on expert system were presented. A rule-based expert system for distributed coordination algorithm was proposed. By using CLIPS and $\mathrm{VC}++$ and other computer language, a combination of computer languages was adopted to implement the control method. The on-line experimental result indicates that, through the collaboration of two expert systems, this control method not only allows robot to fulfill autonomous inspection on transmission line, but also enable the robot to carry out inspection tasks under remote manual control, meanwhile crossing a variety of typical obstacles on the line.

\section{Introduction}

Safe and stable operation of power transmission line is one of the important guarantees for the sustainable development of our economy. Traditional manual inspection is not applicable for all the Extra-High Voltage(EHV) lines due to their complex location distribution. Therefore, replacing manual inspection methods with application of inspection robot technology has become a research hot spot in recent years. Based on study on power transmission line inspection robot, Tokyo Electric Power Company, the United States TRC company as well as the Hydro-Québec's research institute of Canada have carried out related researches ${ }^{[1,2]}$. Domestically, National 863 Program, some major universities and research institutes have also carried out studies on inspection robot since 2002, and have already acquired some progress. However, there are still problems about inspection robot such as its large size and low obstacle crossing efficiency ${ }^{[3,4]}$.

Based on research results of inspection robots both at home and abroad as well as previous study on high-voltage power transmission line inspection robot from Shandong University of Science and Technology, a new mechanical structure of inspection robot on the basis of virtual prototype technology is proposed. It has strong climbing ability and large operation range and is efficient in obstacle crossing, which compensate the above shortcomings to a certain extent. This paper mainly studies the robot's mechanical structure and principle of modern expert intelligent control system, and discusses an intelligent control system software based on expert database and the corresponding hardware implementation method aiming at how it realizes the control function.

\section{Mechanical and Control System Structure of Inspection Robot}

Considering functional requirements of the inspection robot and its work process and environment, the robot uses a three-armed structure, as shown in Fig.1. This robot consists of four parts: the front arm, the mid-arm, the rear arm and the center of gravity regulation box. Both front arm and rear arm are equipped with travelling mechanism, auxiliary wheel and brake apparatus. Mid-arm is installed with travelling mechanism including two travelling wheels and the brake apparatus. All the arms are equipped with telescoping mechanism which allows the arms to extend and shorten as needed, together with two rotation joints in vertical and horizontal direction respectively. The center of gravity regulation box includes the center of gravity regulation mechanism and control box. Center of gravity regulation mechanism moves the control box forward or backward, to regulate the center of gravity of the robot. 


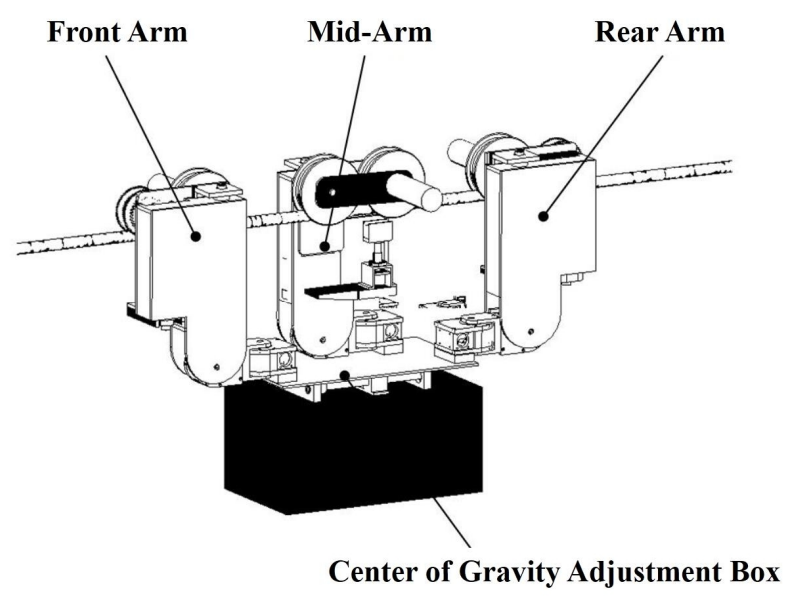

Fig.1 Configuration of Inspection Robot.

The robot can promptly perform obstacle recognition and crossing while walking along the transmission line; in addition, it is able to transmit the environment of transmission line to the ground monitoring system by the main control computer installed in the center of gravity regulation box.

To satisfy the functional requirements, the robot is designed to be capable of working under two different modes: automatic operation mode and remote control mode. These two modes complement and coordinate with each other to realize inspection functions under a variety of conditions. The robot can work on transmission line with fittings like dampers, suspension insulators and clamps under normal condition, and can also perform inspection task under remote manual control in a more complex environment, transmitting information of transmission line to the ground control system. According to the modern control theory and the Hierarchical Intelligent Control theory from G. N. Saridis $^{[5,6,7]}$, a three-level structure for control system is designed, as shown in Fig.2. This system is consisted of ground control system(remote control system), robot main control system(organization level) and lower computer system(execution level).

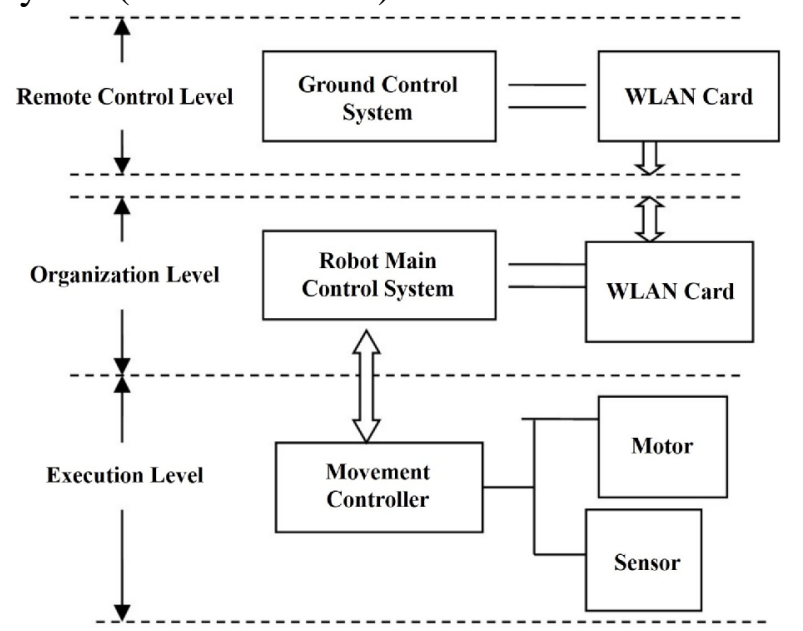

Fig.2 Diagram of Inspection Robot Control System Structure.

According to the overall control system program and hierarchical intelligent control theory, the hardware units of control system is divided into the following three parts:

Remote monitoring system: it is consisted of the monitoring host and wireless remote control module. The monitoring host stores, processes and displays images captured by camera. In addition, the robot can be controlled remotely through a wireless network by the host.

The robot main control system: the main functions are task decomposition and behavior coordination. Under remote control mode, it receives control commands sent by the remote monitoring host, translates them into a motor action sequence, then sends them to the lower computer. Under automatic operation mode, it determines the type of obstacles, robot's position and posture based on the combination of image processing results and sensor feedback, then conducts robot motion planning 
and sends action sequence to the lower computer. Besides, it transmits collected images to remote monitoring host lively over wireless network.

Lower computer: it is able to process sensor information, execute complex logic and arithmetic operations and communicate with the host computer. In addition, it also has motor drive and control function.

\section{Research and Implementation of Expert Control System}

\section{A. Structure of Expert Control System}

Power transmission line inspection robots generally work in harsh environments. They should be able to cross a variety of obstacles, process the images collected by camera and send them to the ground monitoring system in time, which requires an effective control algorithm that insures smooth running of the entire work process. After repeated demonstration studies based on previous research $^{[8,9,10]}$, the expert system which can simulate the expert's analysis, judgement and decision-making process is developed. Its distribution structure is shown in Fig.3.

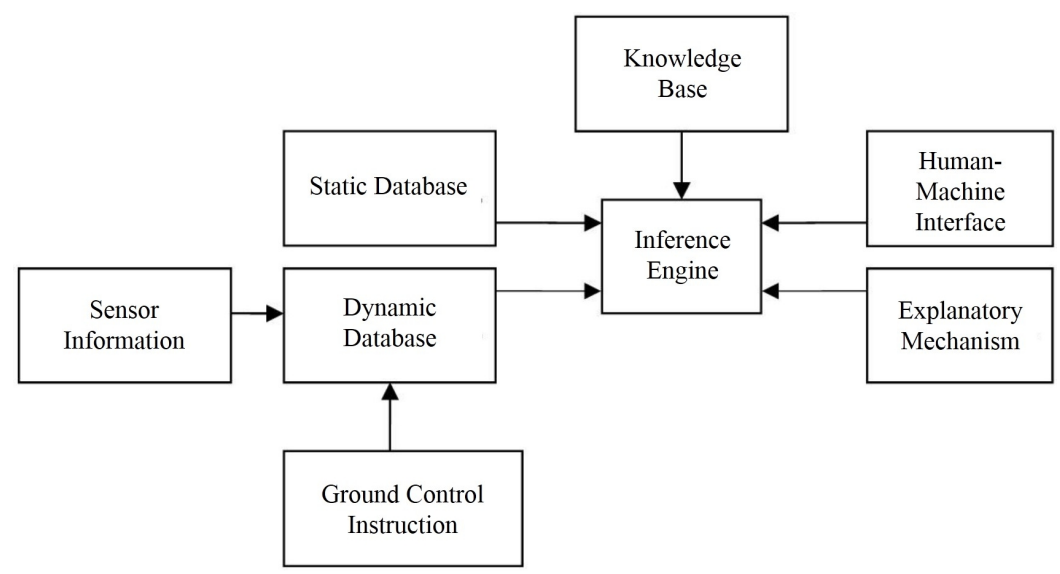

Fig.3 Configuration of Distribute Expert System.

This expert system includes ground monitoring expert system and robot expert system. The robot expert system consists of global dynamic database, static database, inference engine and knowledge base. Information of detection unit and instructions from the ground expert system are stored in dynamic database, while the static database stores the structural parameters of obstacles on $110 \mathrm{kV}$ transmission line. Robotic obstacle crossing behavior is controlled based on the combination of data from dynamic and static database and advices from the knowledge base.

\section{B. CLIPS Embedded and Realization of VC Development Expert Control System Approach}

CLIPS is an expert system development tool designed by NASA in C language, it is a multi-paradigm programming language that supports rules-based, process-oriented and object-oriented programming. CLIPS is portable and is able to be installed on many types of computers. It uses pattern matching algorithm, which takes full advantage of the machine time redundancy, making pattern matching quiet efficient ${ }^{[11,12,13]}$. However, it needs a large amount of storage space to ensure the efficiency of CLIPS, meanwhile its data processing ability is limited, so it is difficult to realize the entire robot control system with only CLIPS. Therefore, $\mathrm{C}$ language, Visual C++ and CLIPS are combined to implement system functions. Since CLIPS is a shareware, and its source code is open to users, we can modify and tailor its kernel according to actual demands. To be specific, the kernel of expert system implemented with CLIPS is implanted into programs wrote in C language and Visual $\mathrm{C}++$.

CLIPS provides a number of method functions with common external program interface. Interface and communication between CLIPS and Visual $\mathrm{C}++$ can be implemented by using these functions. Users can use embedded method and DLL dynamic link library for hybrid programming. 
Dynamic link library (clips.dll) is provided by CLIPS developers. It encapsulates multiple core commands, allows users to embed CLIPS into C++ programs. CCLIPSWrap is defined as a class in header file clipsmfc.h. CLIPS functions can be directly used by means of member variables and member functions from this class. However, it is a common class and is not able to meet the functional demands of this system. To solve this problem, CCLIPSWrap is recompiled, new data members are added, including pointers to cases, disciplines and templates, and functions that be used to obtain cases, disciplines, global variables and agendas.

\section{Distributed Expert System Coordination Algorithm}

Accomplishment of some complex control tasks needs the cooperation of two expert systems, which may bring redundant information in the control system. One expert system also has redundant information due to multiple sensors. So a particular coordination mechanism is designed to solve the issue of information fusion distributed expert system.

Certainty factor(CF), as a measure of the uncertainty of disciplines and facts, is introduced in the inspection robot distributed expert system, the knowledge can be expressed by:

\section{IF A THEN B(CF(B,A)),}

where $A$ is the logic combination of evidence, $C F(B, A)$ is certainty factor, if $C F(B, A)>0$, then conclusion $B$ can be drawn. This knowledge means: conclusion $B$ has certainty factor of $C F(B, A)$ when evidence $\mathrm{A}$ is true. $\mathrm{CF}(\mathrm{B}, \mathrm{A})$ can be calculated by:

$$
C F(B, A)=\left\{\begin{array}{l}
\frac{P(B \mid A)-P(B)}{1-P(B)}, \text { if } P(B \mid A) \geq P(B) \\
\frac{P(B \mid A)-P(B)}{P(B)} \text {, if } P(B \mid A)<P(B)
\end{array},\right.
$$

where $\mathrm{P}(\mathrm{B} \mid \mathrm{A})$ and $\mathrm{P}(\mathrm{B})$ are the priori probability of statistical results. Rules of inspection robot expert system are as follows:

( 1 ) $\mathrm{CF}(\mathrm{B})=\mathrm{CF}(\mathrm{B}, \mathrm{A}) \mathrm{CF}(\mathrm{A})$,

(2) $\mathrm{CF}(\sim \mathrm{A})=-\mathrm{CF}(\mathrm{A})$,

( 3 ) $\mathrm{CF}(\mathrm{A} 1 \mathrm{I} \mathrm{A} 2)=\min (\mathrm{CF}(\mathrm{A} 1), \mathrm{CF}(\mathrm{A} 2)), \mathrm{CF}(\mathrm{A} 1 \cup \mathrm{A} 2)=\max (\mathrm{CF}(\mathrm{A} 1), \mathrm{CF}(\mathrm{A} 2))$,

(4) If the following rules:

IF A1 THEN B(CF(B, A1)),

IF A2 THEN B(CF(B, A2)),

and

$$
\begin{aligned}
& \mathrm{CF} 1(\mathrm{~B})=\mathrm{CF}(\mathrm{B}, \mathrm{A} 1) \mathrm{CF}(\mathrm{A} 1), \\
& \mathrm{CF} 2(\mathrm{~B})=\mathrm{CF}(\mathrm{B}, \mathrm{A} 2) \mathrm{CF}(\mathrm{A} 2),
\end{aligned}
$$

then

$$
C F(B)=\left\{\begin{array}{c}
C F 1(B)+C F 2(B)-C F 1(B) C F 2(B), \text { if } C F 1(B), C F 2(B) \geq 0 \\
C F 1(B)+C F 2(B)+C F 1(B) C F 2(B), \text { if } C F 1(B), C F 2(B)<0, \\
C F 1(B)+C F 2(B), \text { else }
\end{array}\right.
$$

$$
C F(B \mid A)=\left\{\begin{array}{l}
C F(B)+C F(A) C F(B, A)-C F(B) C F(A) C F(B, A), \text { if } C F(B), C F(B, A) \geq 0 \\
C F(B)+C F(A) C F(B, A)(1+C F(B)), \text { if } C F(B), C F(B, A)<0 \\
C F(B)+C F(A) C F(B, A), \text { else }
\end{array} .\right.
$$

The certainty factor of disciplines of expert system is calculated according the rules above, besides information fusion of distributed expert system is accomplished. The strategy for conflict-resolution is:

a) if $\mathrm{CF} \geq 0.99$, conclusions according to the rules above can be drawn, and corresponding operations are adopted; 
b) if $\mathrm{CF}<0.99$, abandon the response;

c) if $0.80<\mathrm{CF}<0.99$, the one with a larger CF wins; when $\mathrm{CF}$ of several disciplines equal, the one with more preconditions wins; and when the number of precondition are still the same, the one ranking in the top wins.

\section{Experimental Analysis}

The transmission line inspection robot with expert control system shows great performance in line inspection, with excellent ability of crossing typical obstacles on simulative transmission line in laboratory environment. Thus the effectiveness of various functions of robot based on the combination of expert database system and on-line signal detection is verified.

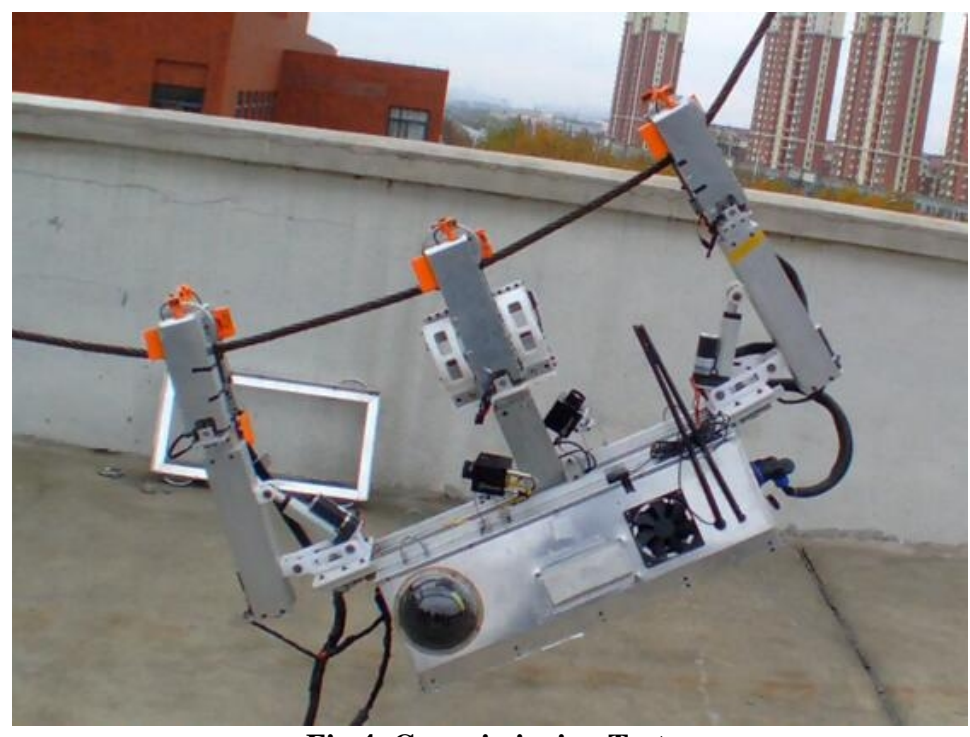

Fig.4 Commissioning Test.

\section{Conclusion and Outlook}

Through in-depth studying of the working principle and mechanical structure of inspection robots, this paper presents a control method based on expert system. Robot can walk along transmission line and autonomously crosses typical obstacles on transmission line with the management and control by this system. Its developed in CLIPS, $\mathrm{C}$ language and Visual $\mathrm{C}++$. There are two expert systems collaborating with each other in the same coordination algorithm. It is tested and verified in laboratory environment that, the design of hardware and software are feasible, and show high reliability and development value especially in terms of robot walking along transmission line.

\section{References}

[1] W. M. Cao: Research on Visual Control Methods for High Voltage Transmission Line Deicing Robot, Hunan University, 2014.

[2] Y. Zhang, Y. Tian, S. Y. Lu, in: Design of a live maintenance mobile robot system for power substation equipment, Chinese Automation Congress (CAC) 2017, pp. 882-887, 2017.

[3] J. Sawada, K. Kusumoto, T. Munakata, et al., in: A Mobile Robot for Inspection of Power Transmission Lines,. IEEE Power Engineering Review, 1991, vol.11(1), pp.57.

[4] Y. C. Zhang, Z. Z. Liang, M. Tan, in: Mobile Robot for Overhead Powerline Inspection - a Review, ROBOT, 2004, vol.26(5), pp.467-473.

[5] G. N. Saridis, Z. Rekasius, in: Design of approximately optimal feedback controllers for systems with bounded states, Automatic Control IEEE Transactions on, vol. 12, pp. 373-379, 1967, ISSN 0018-9286. 
[6] R. T. Kitahara, G. N. Saridis, in: Computational aspects of performance-adaptive self-organizing control algorithms[J]. 1972.

[7] G. N. Saridis: Comparison of five popular identification algorithms: A survey[C], Decision and Control, 1972 and 11th Symposium on Adaptive Processes. Proceedings of the 1972 IEEE Conference on. IEEE, 1972:40-45.

[8] Z. X. Cai, Z. M, Jiang, in: High-Level Robot Planning Based on Expert System, ACTA ELECTRONICA SINICA, 1993, vol.3, pp.88-90.

[9] T. Fukuda, H. Hosokai, M. Otsuka, in: Trajectory Generation and Path Planning Expert System for Inspection Robots[C], IEEE International Workshop on Intelligent Robots. IEEE Xplore, 1988:187-192.

[10] F. Y. Zhou, J. D. Wang, Y. B. Li, et al., in: Control of an inspection robot for 110KV power transmission lines based on expert system design methods[C], Control Applications, 2005. CCA 2005. Proceedings of 2005 IEEE Conference on. IEEE, 2005:1563-1568.

[11] J. Giarratano, G. Riley, Riley, in: Expert Systems[M], Machinery Industry Press, 2005.

[12] H. E. Tang, S. Wen, in: Application of CLIPS in the Engineering Graphics Expert System, Journal of Computer Applications, 2003, vol.23(2), pp.24-26.

[13] X. M. Ye, G. Y. Yuan, in: The Realization of Fault Diagnosis with CLIPS 6 Embedded in C++ Builder 4, Computer Application and Software, 2000, vol.17(10), pp.50-56. 